\title{
SMOOTHNESS OF DECAYING WAVE MOTIONS
}

\section{JEFFREY RAUCH ${ }^{1}$}

ABSTRACT. We show that for compactly supported perturbations of the wave equation, solutions whose total energy decays to zero must be smooth, provided that there are no trapped rays.

This paper discusses solutions to perturbed wave equations where the perturbation is restricted to a compact subset of space. The basic intuitive idea which lies behind the results and proofs is that a solution represents a wave which moves freely except when it lies in the compact set. The expected global behavior is that the wave will stay in this region for a while and then travel off to infinity as a free wave. Since energy is not lost in this last phase, we do not expect that the total energy of a wave will decay to zero as time goes to infinity. In fact, for solutions which are not smooth we can make this argument precise by keeping track of the singularities to show that a finite amount of energy does radiate to infinity. In this way we prove that waves whose total energy decays to zero must be smooth.

Let

$$
L=\frac{\partial^{2}}{\partial t^{2}}-\sum a_{i j}(t, x) \frac{\partial^{2}}{\partial x_{i} \partial x_{j}}+\sum a_{i}(t, x) \frac{\partial}{\partial x_{i}}+a(t, x) \frac{\partial}{\partial t}+b(t, x) .
$$

We suppose that $a_{i j}$ is a positive definite symmetric matrix and that

1. $L=\partial^{2} / \partial t^{2}-\Sigma \partial^{2} / \partial x_{i}^{2}$ for $|x|>R$.

2. The coefficients of $L$ are real valued and smooth.

3. $n$, the number of space dimensions, is odd and greater than 2 .

The reality hypothesis allows us to restrict attention to real solutions and to avoid annoying complex conjugates and real parts. It is not essential. We are interested in solutions $u(t, x)$ of the equation $L u=0$ which have the property that

Received by the editors December 19, 1973.

AMS (MOS) subject classifications (1970). Primary 35L15; Secondary 35L45, 35P25, 35B40, 35D10.

${ }^{1}$ Research partially supported by the National Science Foundation under grant NSF GP 34260. 


$$
E(t) \equiv \int_{\mathrm{R}^{n}} u_{t}^{2}+\left|\nabla_{x} u\right|^{2} d x<\infty \quad \text { for all } t .
$$

The quantity $E(t)$ will be called the energy at time $t$, and classical methods show that if $E(t)<\infty$ for one time $t$, it is finite for all $t$. We further restrict attention to those operators for which "signals" are expected to propagate to infinity. Let $\sigma(t, x, \tau, \xi)=\tau^{2}-\Sigma a_{i j}(t, x) \xi_{i} \xi_{j}$ be the principal symbol of $L$, and let $\chi$ be the vector field

$$
\left(\frac{\partial \sigma}{\partial \tau}, \nabla_{\xi} \sigma,-\frac{\partial \sigma}{\partial t},-\nabla_{x} \sigma\right) \quad \text { on } \mathbf{R} \times \mathbf{R}^{n} \times \mathbf{R} \times\left(\mathbf{R}^{n \backslash 0}\right) \text {. }
$$

A null-bicharacteristic of $L$ is defined as a solution $\gamma(s)$ of the ordinary differential equations $d \gamma / d s=\chi(\gamma(s))$ such that $\sigma(\gamma(s))=0$. The projection of such a curve on $x$-space is called a ray, and we say that a point on the curve lies over $x_{0}$ if its projection is $x_{0}$.

On a null-bicharacteristic we have $d t / d s=\partial \sigma / \partial \tau=2 \tau \neq 0$ so that $t$ can be introduced as a parameter on any ray. It $x(t)$ is a ray described in this fashion we say that $x(t)$ leaves the set $\omega$ at time $t_{0}$ if $x\left(t_{0}\right) \in \omega$ and there exist $t_{n}>t_{0}$ with $t_{n} \rightarrow t_{0}$ and $x\left(t_{n}\right) \notin \omega$. The next, and last, hypothesis on $L$ is crucial.

4.

Any ray which intersects the set $\omega=\{x|| x \mid \leq R\}$

leaves $\omega$ at some finite time. That is, there are no rays trapped in $\omega$ as $t \rightarrow+\infty$.

Because of hypothesis 4, the behavior of null-bicharacteristics as $t \rightarrow+\infty$ is quite simple. Suppose that $\gamma(s)=(t(s), x(s), \tau(s), \xi(s))$ is such a bicharacteristic. Suppose $d t / d s>0$ so that $t$ increases when $s$ increases ( $d t / d s<0$ is similar). If $|x(s)|>R$ for all $s$, then $\gamma$ is just a null-bicharacteristic for the free wave equation. If $\gamma$ passes over the ball of radius $R$, then we may choose $s_{0}$ so that the associated ray leaves $\omega$ at time $t\left(s_{0}\right)$. Then $\left|x\left(s_{0}\right)\right|=R$ and

$$
0 \leq \frac{d}{d s}|x|^{2}\left(s_{0}\right)=2 x\left(s_{0}\right) \frac{d x}{d s}\left(s_{0}\right)=4 x\left(s_{0}\right) \cdot \xi\left(s_{0}\right) .
$$

An elementary computation shows that for $s \geq s_{0}$

$$
\gamma(s)=\left(t\left(s_{0}\right)+2 s r\left(s_{0}\right), x\left(s_{0}\right)+2 s \xi\left(s_{0}\right), r\left(s_{0}\right), \xi\left(s_{0}\right)\right) .
$$

The corresponding ray is a straight line traveling from the sphere of radius $R$ to infinity in the direction $\xi\left(s_{0}\right)$. To summarize: every ray eventually travels to infinity as a straight line. 
Main Theorem. If $L$ is a wave operator satisfying conditions one to four and $u$ is a solution of $L u=0$ with $0=\lim _{t \rightarrow+\infty} \int_{\mathbb{R} n}\left(u_{t}^{2}+\left|\nabla_{x} u\right|^{2}\right)$, then $u \in C^{\infty}\left(\mathbf{R} \times \mathbf{R}^{n}\right)$.

The proof uses two powerful tools; the incoming and outgoing spaces $D_{ \pm}$of Lax and Phillips, and Hörmander's theorem on the propagation of singularities. First we describe the relevant facts about $D_{ \pm^{*}}$

Let $L_{0}=\partial^{2} / \partial t^{2}-\Delta$ and $\mathcal{U}(t)$ the operator defined by $\mathcal{U}(t)(\phi, \psi)=$ $\left(v(t), v_{t}(t)\right)$, where $v$ is the solution of $L_{0} v=0, v(0)=\phi, v_{t}(0)=\psi$. Let $\mathcal{H}$ be the completion of $C_{0}^{\infty}\left(\mathbf{R}^{n}\right) \times C_{0}^{\infty}\left(\mathbf{R}^{n}\right)$ in the "energy norm"

$$
|(\phi, \psi)|^{2}=\int_{R^{n}}\left(|\nabla \phi|^{2}+\psi^{2}\right) \text {. }
$$

$\mathcal{U}(t)$ is then a strongly continuous unitary group on the Hilbert space $\mathcal{H}$.

Definition. $D_{+}$(resp. $\left.D_{-}\right)=\{\Phi \in \mathcal{H} \mid \mathcal{U}(t) \Phi=0$ for $|x|<t$ (resp. $\left.|x|<-t)\right\}$.

Aside from elementary properties of $D_{ \pm}$we need the deep fact that for $n$ odd, $D_{+}$and $D_{\text {_ }}$ are orthogonal complements in $\mathcal{H}$. The connection with Hörmander's theorem is illustrated by the following result which does not depend on the parity of $n$.

Lemma 1. Suppose that $u$ is a finite energy solution of the free wave equation $u_{t t}=\Delta u$ and that $(0, x,|x|, x) \in W F(u)$ for some $x \neq 0$, then $\left(u(0), u_{t}(0)\right) \notin D_{-}$.

Proof. The important property of $(0, x,|x|, x)$ is that $x$ and $\xi$ components point in the same direction. The null-bicharacteristic of $L_{0}$ through this point is $\gamma(s)=(2|x| s, x+2 s x,|x|, x)$ and the corresponding ray travels radially to infinity as $s$, and therefore $t$, approaches $+\infty$. Hörmander's theorem implies that the entire bicharacteristic lies in the wavefront set of $u$. For $s=-1 / 2$ we see that $(-|x|, 0,|x|, x) \in W F(u)$ so $u$ does not vanish in any neighborhood of $(-|x|, 0)$ so $u \notin D_{-}$.

The fact that $(-|x|, 0,|x|, x) \in W F(u)$, implies the stronger assertion that $\left(u(-t), u_{t}(-t)\right) \notin D_{-}$for all $t<|x|$. To use this result we make a few alterations.

Definitions. $D_{+}^{\rho}=\mathcal{U}(\rho) D_{+}, \pi_{+}^{\rho}=$ orthogonal projection (in $\mathcal{H}$ ) on the subspace $D_{+}^{\rho}$

The remark following the proof of Lemma 1 with the fact that for $n$ odd $D_{+}$and $D_{-}$are orthogonal complements proves

Lemma 2. If $n$ is odd and the hypotheses of Lemma 1 hold, then $\pi_{+}^{P}\left(u(0), u_{t}(0)\right) \neq 0$ provided $\rho<|x|$. 
We need to consider states which are outgaing with respect to points other than the origin.

Definitions. $D_{+}\left(x_{0}\right)=\left\{\Phi \in \mathcal{H} \mid \mathcal{U}(t) \Phi=0\right.$ for $\left.\left|x-x_{0}\right|<t\right\} . D_{+}^{\rho}\left(x_{0}\right)=$ $\mathcal{U}(\rho) D_{+}\left(x_{0}\right) \cdot \pi_{+}^{\rho}\left(x_{0}\right)=$ orthogonal projection (in $\left.\mathcal{H}\right)$ on $D_{+}^{\rho}\left(x_{0}\right)$.

The generalization of Lemma 2 to these spaces presents no new difficulties. The advantage of the new spaces is that a typical outgoing ray will not be traveling directly away from the origin but will be traveling radially with respect to some new center $x_{0}$.

Proof of the main theorem. Suppose that $u$ is a finite energy solution of $L u=0$ and that $u \notin C^{\infty}\left(\mathbf{R} \times \mathbf{R}^{n}\right)$. We will show that $\lim \inf _{t \rightarrow \infty} E(t)>0$. By hypothesis there is a point $P_{0}=\left(t_{0}, x_{0}, \tau_{0}, \xi_{0}\right) \in W F(u)$. It follows that $\sigma\left(P_{0}\right)=0$ and that the entire null-bicharacteristic, $\gamma(s)$, of $L$ with $\gamma(0)=$ $P_{0}$ must lie in $W F(u)$. As usual, either $d t / d s>0$ or $d t / d s<0$ for all $s$. Since both cases can be handled by essentially the same argument, we may suppose that $d t / d s>0$. We then know that there is an $s_{1}$ such that for $s \geq s_{1}, \gamma(s)=P_{1}+2 s\left(\tau_{1}, \xi_{1}, 0,0\right)$, where $P_{1}=\gamma\left(s_{1}\right)=\left(t_{1}, x_{1}, \tau_{1}, \xi_{1}\right)$, $\left|x_{1}\right|=R, x_{1} \cdot \xi_{1} \geq 0$. It follows from the generalization of Lemma 2 that

$$
\pi_{+}^{0}\left(x_{1}\right)\left(u(t), u_{t}(t)\right) \neq 0 \text { for all } t>t_{1} .
$$

Choose $T$ so large that $|x| \leq R$ implies $2\left|x-x_{1}\right| \leq\left(T-t_{1}\right)$. Let $\rho=$ $\left(T-t_{1}\right) / 2$ and $\Phi=\left(u(T), u_{t}(T)\right) \in \mathcal{H}$. The fact $(*)$ implies that $\pi_{+}^{\rho}\left(x_{0}\right) \Phi \neq$

0 . Shifting the time scale if necessary we may assume that $T=0$.

With this convention, notice that for $\left|x-x_{1}\right|>t+\rho \geq \rho$, we have $u=$ $v$, where $v$ is the solution of $v_{t t}=\Delta v$ on $\mathbf{R} \times \mathbf{R}^{n},\left(v(0), v_{t}(0)\right)=\Phi$. Recall that $\mathrm{U}(t)$ is defined so that $\left(\nu(t), v_{t}(t)\right)=\mathcal{U}(t) \Phi$. We need to evaluate the norm and scalar product in $\mathcal{H}$ over subsets of $\mathbf{R}^{n}$. For $\beta \subset \mathbf{R}^{n}, \Phi=\left(\phi_{1}, \phi_{2}\right), \Psi=$ $\left(\psi_{1}, \psi_{2}\right)$ in $\mathcal{H}$ let

$$
(\Phi, \Psi)_{\beta}=\int_{\beta}\left(\nabla \phi_{1} \cdot \nabla \psi_{1}+\psi_{2} \psi_{2}\right), \quad|\Phi|_{\beta}=(\Phi, \Phi)_{\beta}^{1 / 2}
$$

Then as $t \rightarrow+\infty$ we have

$$
\begin{aligned}
\lim \inf \left|\left(u(t), u_{t}(t)\right)\right| & \geq \lim \inf \left|\left(v(t), v_{t}(t)\right)\right|_{\left|x-x_{1}\right|>t+\rho} \\
& =\lim \inf |U(t) \Phi|_{\left|x-x_{1}\right|>t+\rho^{\circ}}
\end{aligned}
$$

Let $\Phi_{+}=\pi_{+}^{\rho}\left(x_{1}\right) \Phi \neq 0$. The proof is completed by showing that for all $t \geq$ $0,|\mathcal{U}(t) \Phi|_{\left|x-x_{1}\right|>t+\rho} \geq\left|\Phi_{+}\right|_{R^{n}}$. To see this let $\Phi_{-}=\Phi-\Phi_{+}$. Then $\Phi_{+}$ 
is orthogonal to $\Phi_{-}$in $\mathcal{H}$ since $\pi_{+}^{\rho}$ is an orthogonal projection, and as a consequence $\mathcal{U}(t) \Phi_{+} \perp \mathcal{U}(t) \Phi_{-}$for all $t$. For fixed $t \geq 0$ let $\mathcal{O}=\left\{x|| x-x_{1} \mid\right.$ $>t+\rho\}$, then

$$
\begin{aligned}
|\mathrm{U}(t) \Phi|_{\mathcal{O}}^{2} & =\left|\mathrm{U}(t) \Phi_{+}+\mathrm{U}(t) \Phi_{-}\right|_{\mathcal{O}}^{2} \\
& =\left|\mathrm{U}(t) \Phi_{+}\right|_{\mathcal{O}}^{2}+|\mathrm{U}(t) \Phi|_{\mathcal{O}}^{2}+2\left(\mathrm{U}(t) \Phi_{+}, \mathrm{U}(t) \Phi_{-}\right)_{\mathcal{O}} .
\end{aligned}
$$

Now $\mathcal{U}(t) \Phi_{+}=0$ on $\mathbf{R}^{n} \backslash \mathcal{O}$ so that $\mathcal{O}$ may be replaced by $\mathbf{R}^{n}$ in the last term so this term vanishes. For the same reason $\left|\mathcal{U}(t) \Phi_{+}\right|_{\mathcal{O}}=\left|\mathcal{U}(t) \Phi_{+}\right|_{\mathbb{R}^{n}}=\left|\Phi_{+}\right|_{\mathrm{R}^{n}}$ and the proof that $|U(t) \Phi|_{\mathcal{O}} \geq\left|\Phi_{+}\right|_{R^{n}}$ is complete.

The proof given above uses in an essential way the facts that $n$ is odd and that the equation $u_{t t}-\Delta u=0$ has precisely one sound speed in each direction. The latter restriction precludes the extension to general systems. In a forthcoming paper [3], M. Taylor and the author overcome these obstacles. For the first we show that for any $n,\left(D_{+}\right)^{\perp}$ consists of functions smooth in the backward light cone and this is precisely what is needed in Lemma 2. The second problem is handled by finding a different way to use the information $\pi_{+}^{\mathcal{P}}\left(x_{1}\right) \Phi \neq 0$. In addition we show that, even in the presence of boundaries, if $u$ is as in the main theorem then $\left(u(t), u_{t}(t)\right)$ cannot have compact support for any $t$.

\section{REFERENCES}

1. P. D. Lax and R. S. Phillips, Scattering theory, Academic Press, New York, 1967.

2. L. Hörmander, On the existence and regularity of solutions of linear pseudodifferential equations, Enseigement Math. 17 (1971), 99-163.

3. J. Rauch and M. Taylor, Decaying states of perturbed wave motion (to appear).

DEP ARTMENT OF MATHEMATICS, UNIVERSITY OF MICHIGAN, ANN ARBOR, MICHIGAN 48104 